\title{
Uncertainty quantification in the damage assessment of a seven-story RC building slice through Bayesian FE model updating
}

\author{
E. Simoen \\ KU Leuven, Dept. of Civil Engrg., Kasteelpark Arenberg 40 box 2448, B-3001 Leuven, Belgium
}

B. Moaveni

Tufts University, Dept. of Civil and Environmental Engrg., 200 College Ave., Medford, MA 02155, USA

J.P. Conte

University of California at San Diego, Dept. of Structural Engrg., 9500 Gilman Dr., La Jolla, CA 92093-0085, USA

G. Lombaert

KU Leuven, Dept. of Civil Engrg., Kasteelpark Arenberg 40 box 2448, B-3001 Leuven, Belgium

\begin{abstract}
Bayesian finite element (FE) model updating is used for uncertainty quantification (UQ) purposes in the vibration-based damage assessment of a seven-story reinforced concrete building slice. This structure was built and tested at full scale on the USCD-NEES shake table: a progressive damage pattern was induced by subjecting the structure to a number of historical earthquake records. At each damage stage, modal characteristics (i.e. natural frequencies and mode shapes) were determined through vibration testing, these data are used in the Bayesian FE model updating schemes. In order to analyze the results of the Bayesian scheme and gain insight into what information is contained in the data, a comprehensive uncertainty and resolution analysis performed. It is shown that the Bayesian UQ approach and subsequent resolution analysis are effective in assessing uncertainty in FE model updating. Furthermore, it is demonstrated that insight into the Bayesian FE model updating approach provides a very natural way to regularize its often ill-posed deterministic counterpart.
\end{abstract}

\section{THE SEVEN-STORY TEST STRUCTURE}

The seven-story test structure (Moaveni et al. 2007, Moaveni et al. 2009, Moaveni et al. 2010, Moaveni et al. 2011) represents a slice of a prototype reinforced concrete mid-rise residential building and is shown in Figure 1a. The structure consists of two perpendicular walls (i.e. a main wall and a back wall for transverse stability), seven concrete floor slabs, an auxiliary post-tensioned column for torsional stability, and four gravity columns to transfer the weight of the slabs to the ground level (Figure 1b).

A progressive damage pattern was induced in the test structure through four historical earthquake records, leading to 5 damage states S0 to S4 (Table 1). After each seismic excitation sequence, vibration tests were performed to obtain modal characteristics of the structure. The experimentally identified natural frequencies of the first three longitudinal modes are listed in Table 2 for each damage state; it is clear that, as expected, the values decrease as the damage increases. Figure 2 shows the corresponding employed mode shapes obtained at damage state S0 using 28 sensors located along the main wall and on the floor slabs. For the damage identification, only the mode shape measurements obtained using 14 sensors located along the main wall are employed. In the following, experimental eigenvalues and mode shapes are denoted as $\tilde{\lambda}_{r}=\left(2 \pi f_{\exp , r}\right)^{2}$ and $\tilde{\phi}_{r} \in \mathbb{R}^{N_{o}}$, respectively, where $N_{o}$ represents the number of observed degrees of freedom. Both experimental data are collected in the vector $\tilde{\mathbf{d}}=\left\{\ldots, \tilde{\lambda}_{r}, \ldots, \tilde{\boldsymbol{\phi}}_{r}^{\mathrm{T}}, \ldots\right\}^{\mathrm{T}}$.

These modal data are used in five consecutive damage analyses: for each damage state, Bayesian FE

Table 1: The five damage states and corresponding imposed historical earthquake records.

\begin{tabular}{c|cccc}
\hline \hline Damage & \multicolumn{4}{|c}{ Earthquake record } \\
state & Earthquake & Comp. & Recorded at & M \\
\hline S0 & None & - & - & - \\
S1 & 1971 S. Fernando & long. & Van Nuys & 6.6 \\
S2 & 1971 S. Fernando & trans. & Van Nuys & 6.6 \\
S3 & 1994 Northridge & long. & Oxnard Blvd & 6.7 \\
S4 & 1994 Northridge & $360^{\circ}$ & Oxnard Blvd & 6.7 \\
\hline \hline
\end{tabular}




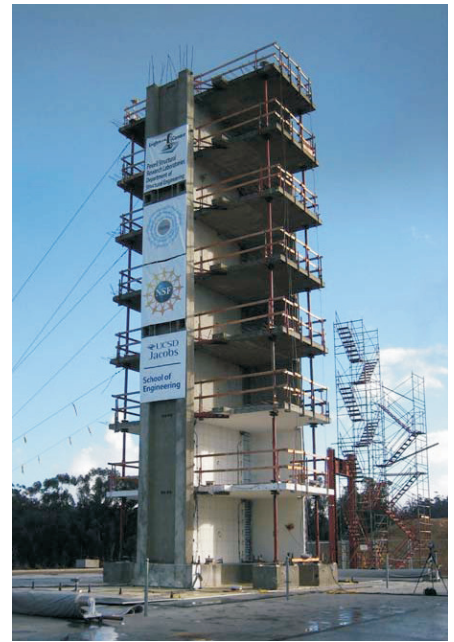

(a)

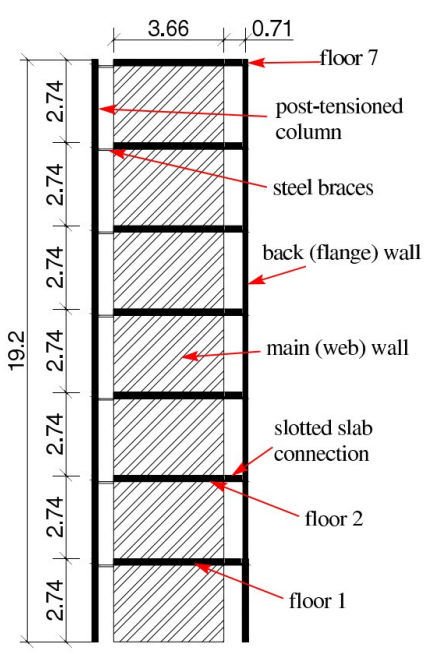

(b)
Figure 1: (a) Seven-story test structure and (b) elevation view.

Table 2: Experimentally identified natural frequencies and damping ratios for the five damage states.

\begin{tabular}{c|ccc}
\hline \hline $\begin{array}{c}\text { Damage } \\
\text { state }\end{array}$ & Mode 1 & $\begin{array}{c}f_{\text {exp }}[\mathrm{Hz}] \\
\text { Mode 2 }\end{array}$ & Mode 3 \\
\hline S0 & 1.91 & 10.51 & 24.51 \\
S1 & 1.88 & 10.21 & 24.31 \\
S2 & 1.67 & 10.16 & 22.60 \\
S3 & 1.44 & 9.23 & 21.82 \\
S4 & 1.02 & 5.67 & 15.10 \\
\hline \hline
\end{tabular}

model updating is applied to quantify the uncertainties on the damage identification results. To this end, a detailed 3D linear elastic FE model was constructed with 322 shell and truss elements and $N_{d}=2418$ degrees of freedom (Figure 3a), using the generalpurpose FE analysis program FEDEASLab (Filippou and Constantinides 2004). In order to model the damage, the structure is divided into 10 substructures, each consisting of part of the main wall (Figure $3 \mathrm{~b})$. It is assumed that each substructure has a uniform effective stiffness (Young's modulus); these stiffness values will be the updating parameters $\boldsymbol{\theta}_{\mathrm{M}}$ in the Bayesian updating schemes. Initial values $\boldsymbol{\theta}_{\mathrm{M}}^{\text {init }}$ of the 10 Young's moduli are obtained trough concrete cylinder testing at various heights along the building (Moaveni et al. 2010):

$$
\begin{aligned}
& \boldsymbol{\theta}_{\mathrm{M}}^{\text {init }}=\left[\begin{array}{lllll}
24.5 & 24.5 & 26.0 & 26.0 & 34.8 \\
34.8 & 30.2 & 28.9 & 32.1 & 33.5
\end{array}\right]^{\mathrm{T}} \quad \mathrm{GPa}
\end{aligned}
$$

The FE model allows for the computation of the modal data as a function of the model parameters $\boldsymbol{\theta}_{\mathrm{M}}$, where the modal data consist of $N_{m}$ eigenvalues $\lambda_{r}$ and corresponding mode shapes $\phi_{r} \in \mathbb{R}^{N_{d}}$, which are the solutions of the (undamped) eigenvalue equation $\mathbf{K}\left(\boldsymbol{\theta}_{\mathrm{M}}\right) \boldsymbol{\Phi}=\mathbf{M} \boldsymbol{\Phi} \boldsymbol{\Lambda}$, where $\mathbf{K}\left(\boldsymbol{\theta}_{\mathrm{M}}\right)$ is the FE model stiffness matrix and $\mathbf{M}$ the mass matrix. $\Phi$ collects the eigenvectors $\phi_{r}$ corresponding to the eigenvalues $\lambda_{r}$ located on the diagonal of $\boldsymbol{\Lambda}$. In the Bayesian updating scheme, these computed modes are paired to

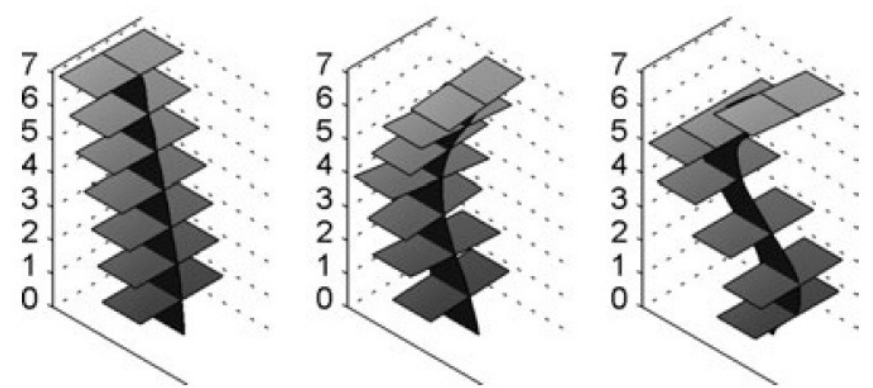

Figure 2: First three longitudinal mode shapes obtained at damage state S0.

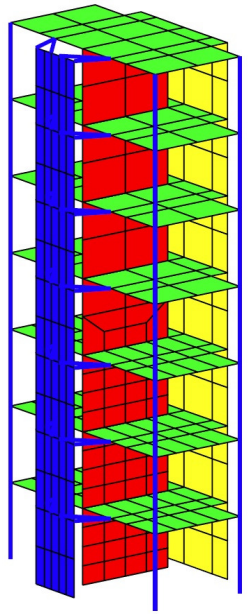

(a)

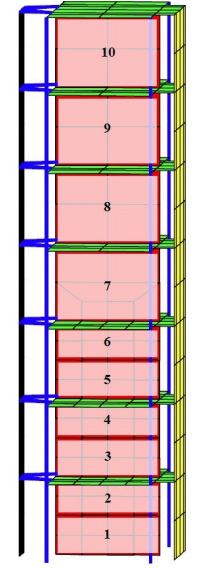

(b)
Figure 3: (a) FE model of the seven-story test structure and (b) definition of the substructures along the main wall.

the experimentally identified modes by means of the Modal Assurance Criterion (or MAC); furthermore, a least squares scaling factor is introduced in order to ensure that paired modes are scaled equally. The set of computed data for a certain model parameter set $\boldsymbol{\theta}_{\mathrm{M}}$ is referred to as $\mathrm{G}_{\mathrm{M}}\left(\boldsymbol{\theta}_{\mathrm{M}}\right)$ in the following.

\section{BAYESIAN FE MODEL UPDATING}

\subsection{Model classes and uncertainties}

In general terms, a model $\mathcal{M}_{\mathrm{M}}\left(\boldsymbol{\theta}_{\mathrm{M}}\right)$ belonging to the model class $\mathcal{M}_{\mathrm{M}}$ provides a mapping from the parameters $\boldsymbol{\theta}_{\mathrm{M}}$ to an output vector $\mathrm{G}_{\mathrm{M}}\left(\boldsymbol{\theta}_{\mathrm{M}}\right) \in \mathbb{R}^{N}$ through the transfer operator $\mathbf{G}_{\mathrm{M}}$. In the ideal case, the model output $\mathrm{G}_{\mathrm{M}}\left(\boldsymbol{\theta}_{\mathrm{M}}\right)$ corresponds perfectly to the true system output $\mathbf{d}$, i.e. $\mathbf{G}_{\mathrm{M}}\left(\boldsymbol{\theta}_{\mathrm{M}}\right)=\mathrm{d}$. This is the main starting point for deterministic parameter identification, where the objective is to determine the model parameters $\boldsymbol{\theta}_{\mathrm{M}}$ for a given set of observed system outputs $\mathbf{d}$. However, the equality $\mathrm{G}_{\mathrm{M}}\left(\boldsymbol{\theta}_{\mathrm{M}}\right)=\mathbf{d}$ is only valid when it is assumed that the underlying fundamental physics of the system are fully known. This is of course never the case, as no model is capable of perfectly representing the behavior of the true physical system. A modeling error $\boldsymbol{\eta}_{\mathrm{G}}$ is therefore always present, and can be described as the discrepancy between the model predictions $\mathrm{G}_{\mathrm{M}}\left(\boldsymbol{\theta}_{\mathrm{M}}\right)$ and the true 
system output d, i.e. $\boldsymbol{\eta}_{\mathrm{G}}=\mathrm{d}-\mathrm{G}_{\mathrm{M}}\left(\boldsymbol{\theta}_{\mathrm{M}}\right)$.

As the true system output has to be measured and processed experimentally, the data $\mathbf{d}$ are always subject to measurement error, resulting in a discrepancy between the true system output $\mathbf{d}$ and the actually observed data $\tilde{\mathrm{d}}$. This difference is defined as the measurement error $\eta_{\mathrm{D}}=\tilde{\mathrm{d}}-\mathrm{d}$. Eliminating the unknown true system output $\mathbf{d}$ from the error equations and collecting both errors on the right hand side of the equation yields:

$\tilde{\mathbf{d}}-\mathrm{G}_{\mathrm{M}}\left(\boldsymbol{\theta}_{\mathrm{M}}\right)=\boldsymbol{\eta}_{\mathrm{G}}+\boldsymbol{\eta}_{\mathrm{D}}=\boldsymbol{\eta}$

The sum of both errors is equal to the total observed prediction error $\boldsymbol{\eta}$, defined as the difference between the observed and predicted response quantities. This expression serves as a starting point for the Bayesian uncertainty quantification method.

\subsection{Bayesian inference methodology}

The general principle behind Bayesian model updating is that the structural model parameters $\boldsymbol{\theta}_{\mathrm{M}} \in \mathbb{R}^{N_{M}}$ that parametrize model class $\mathcal{M}_{\mathrm{M}}$ are modeled as random variables, i.e. probability density functions (PDFs) are assigned to these parameters, which are then updated in the inference scheme based on the available information. Measurement and modeling uncertainty are taken into account by modeling the respective errors as random variables as well: PDFs are assigned to $\boldsymbol{\eta}_{\mathrm{G}}$ and $\boldsymbol{\eta}_{\mathrm{D}}$, which are parametrized by parameters $\boldsymbol{\theta}_{\mathrm{G}} \in \mathbb{R}^{N_{G}}$ and $\boldsymbol{\theta}_{\mathrm{D}} \in \mathbb{R}^{N_{D}}$. These parameters are added to the structural model parameters $\boldsymbol{\theta}_{\mathrm{M}}$ to form the general model parameter set $\boldsymbol{\theta}=\left\{\boldsymbol{\theta}_{\mathrm{M}}, \boldsymbol{\theta}_{\mathrm{G}}, \boldsymbol{\theta}_{\mathrm{D}}\right\}^{\mathrm{T}} \in \mathbb{R}^{N}$. This in fact corresponds to adding two probabilistic model classes to the structural model class $\mathcal{M}_{\mathrm{M}}$ to form a joint model class $\mathcal{M}=\mathcal{M}_{\mathrm{M}} \times \mathcal{M}_{\mathrm{G}} \times \mathcal{M}_{\mathrm{D}}$, parametrized by $\boldsymbol{\theta}$.

To express the updated joint PDF of the unknown parameters $\boldsymbol{\theta}$, given some observations $\tilde{\mathrm{d}}$ and a certain joint model class $\mathcal{M}$, Bayes' theorem is used:

$p(\boldsymbol{\theta} \mid \tilde{\mathbf{d}}, \mathcal{M})=c p(\tilde{\mathbf{d}} \mid \boldsymbol{\theta}, \mathcal{M}) p(\boldsymbol{\theta} \mid \mathcal{M})$

where $p(\boldsymbol{\theta} \mid \tilde{\mathbf{d}}, \mathcal{M})$ is the updated or posterior joint PDF of the model parameters given the measured data $\tilde{\mathrm{d}}$ and the assumed model class $\mathcal{M}$; $c$ is a normalizing constant (independent of $\boldsymbol{\theta}$ ) that ensures that the posterior PDF integrates to one; $p(\tilde{\mathbf{d}} \mid \boldsymbol{\theta}, \mathcal{M})$ is the PDF of the observed data given the parameters $\boldsymbol{\theta}$; and $p(\boldsymbol{\theta} \mid \mathcal{M})$ is the initial or prior joint PDF of the parameters. In the following, the explicit dependence on the model class $\mathcal{M}$ is omitted in order to simplify the notations.

\subsection{Prior $P D F$}

The prior PDF $p(\boldsymbol{\theta})$ represents the probability distribution of the model parameters $\boldsymbol{\theta}$ in the absence of observations or measurement results. In most cases, this
PDF is chosen based on engineering judgment and the available prior information; alternatively, the Principle of Maximum Entropy (Jaynes 1957, Soize 2003, Soize 2008) provides an objective method to determine suitable prior PDFs that yield maximum uncertainty given the available information.

\subsection{Likelihood function}

The PDF of the experimental data $p(\tilde{\mathbf{d}} \mid \boldsymbol{\theta})$, also referred to as the likelihood function $L(\boldsymbol{\theta} \mid \tilde{\mathbf{d}})$, can be interpreted as a measure of how good a model succeeds in explaining the observations $\tilde{\mathbf{d}}$. It reflects the contribution of the measured data $\tilde{\mathbf{d}}$ in the determination of the updated PDF of the model parameters $\boldsymbol{\theta}$, and may be determined according to the Total Probability Theorem and Eq. (2) using the probabilistic models of the measurement and modeling errors:

$$
\begin{aligned}
L(\boldsymbol{\theta} \mid \tilde{\mathbf{d}}) \equiv & p(\tilde{\mathbf{d}} \mid \boldsymbol{\theta})=\int p_{\boldsymbol{\eta}_{\mathrm{D}}}\left(\mathbf{d}-\tilde{\mathbf{d}} \mid \boldsymbol{\theta}_{\mathrm{D}}\right) \\
& \times p_{\boldsymbol{\eta}_{\mathrm{G}}}\left(\mathbf{G}_{\mathbf{M}}\left(\boldsymbol{\theta}_{\mathrm{M}}\right)-\mathbf{d} \mid \boldsymbol{\theta}_{\mathrm{G}}\right) d \mathbf{d}
\end{aligned}
$$

This equation shows that the likelihood function can be computed as the convolution of the PDFs of the measurement and modeling error. When no information is available on the individual errors, as is most often the case, the likelihood function can be constructed using the probabilistic model of the total prediction error $\boldsymbol{\eta}$ parameterized by $\boldsymbol{\theta}_{\eta}$ :

$L(\boldsymbol{\theta} \mid \tilde{\mathbf{d}}) \equiv p(\tilde{\mathbf{d}} \mid \boldsymbol{\theta})=p\left(\boldsymbol{\eta} \mid \boldsymbol{\theta}_{\eta}\right)$

Usually, the prediction error model is assumed fixed (reducing the parameter set to $\boldsymbol{\theta}=\boldsymbol{\theta}_{\mathrm{M}}$ ) and Gaussian.

\subsection{Posterior PDF}

When the prior PDF and likelihood function are determined, Eq. (3) allows for the updating of the joint PDF of the model parameters $\boldsymbol{\theta}$ based on experimental observations of the system. For most practical applications where multiple parameters are involved, computing the posterior joint and marginal PDFs requires solving high-dimensional integrals. Therefore use is often made of asymptotic expressions (Beck and Katafygiotis 1998, Papadimitriou et al. 1997) or sampling methods such as Markov Chain Monte Carlo (MCMC) methods (Gamerman 1997) and its derivatives, e.g. Delayed-Rejection Adaptive Metropolis-Hastings MCMC (Haario et al. 2001, Haario et al. 2006) and Transitional MCMC (Ching and Chen 2007).

\subsection{Bayesian FE model updating of the seven-story test structure}

To quantify the uncertainties in the multi-stage damage assessment of the seven-story building slice intro- 
duced above, the Bayesian inference method elaborated above is applied to this test case. For each of the considered damage states, a Bayesian updating routine is performed, using the following scheme:

S0: The initial values $\boldsymbol{\theta}^{\text {init }}$ in Eq. (1) are adopted as most probable prior point or Maximum A Priori (MAPr) estimate of the model parameters $\boldsymbol{\theta}$. The prior $\operatorname{PDF} p\left(\boldsymbol{\theta} ; \boldsymbol{\theta}^{\text {init }}\right)$ is updated to a posterior PDF $p\left(\boldsymbol{\theta} \mid \tilde{\mathbf{d}}^{(S 0)}\right)$ through the likelihood function $L\left(\boldsymbol{\theta} \mid \tilde{\mathbf{d}}^{(S 0)}\right)$, which is constructed using the measured modal data $\tilde{\mathbf{d}}^{(S 0)}$ obtained in damage state S0:

$p\left(\boldsymbol{\theta} \mid \tilde{\mathbf{d}}^{(S 0)}\right) \propto p\left(\boldsymbol{\theta} ; \boldsymbol{\theta}^{\text {init }}\right) L\left(\boldsymbol{\theta} \mid \tilde{\mathbf{d}}^{(S 0)}\right)$

$\mathrm{S} 1$ : In the next stage $\mathrm{S} 1$, the MAP estimate $\hat{\boldsymbol{\theta}}_{\mathrm{MAP}}^{(S 0)} \mathrm{ob}-$ tained in $\mathrm{S} 0$ is adopted as maximum a priori estimate in the prior PDF of S1. To obtain the posterior PDF for this stage, the prior has to be multiplied with the likelihood function $L\left(\boldsymbol{\theta} \mid \tilde{\mathbf{d}}^{(S 1)}\right)$ which is constructed using modal data obtained in $\mathrm{S} 1$ :

$p\left(\boldsymbol{\theta} \mid \tilde{\mathbf{d}}^{(S 1)}\right) \propto p\left(\boldsymbol{\theta} ; \hat{\boldsymbol{\theta}}_{\mathrm{MAP}}^{(S 0)} L\left(\boldsymbol{\theta} \mid \tilde{\mathbf{d}}^{(S 1)}\right)\right.$

$\mathrm{S} k$ : This scheme is repeated for the next stages, such that for an arbitrary stage $S k$ the following updating equation is obtained:

$p\left(\boldsymbol{\theta} \mid \tilde{\mathbf{d}}^{(S k)}\right) \propto p\left(\boldsymbol{\theta} ; \hat{\boldsymbol{\theta}}_{\mathrm{MAP}}^{(S(k-1))}\right) L\left(\boldsymbol{\theta} \mid \tilde{\mathbf{d}}^{(S k)}\right)$

\subsubsection{Prior PDF}

The joint prior PDF for the model parameters $\boldsymbol{\theta}$ is determined based on the Maximum Entropy Principle (Soize 2003, Soize 2008), which can be shown to lead to a priori independent Gamma-distributed variables. This means the joint prior PDF for the first undamaged stage $\mathrm{S} 0$ is given as:

$p\left(\boldsymbol{\theta} ; \boldsymbol{\theta}^{\mathrm{init}}\right)=\prod_{j=1}^{N_{\theta}} \frac{\theta_{j}^{\alpha_{j}-1}}{\beta_{j}^{\alpha_{j}} \Gamma\left(\alpha_{j}\right)} \exp \left(-\frac{\theta_{j}}{\beta_{j}}\right)$

where shape factor $\alpha_{j}\left(=\mu_{j}^{2} / \sigma_{j}^{2}=1 / \mathrm{COV}_{j}^{2}\right)$ and scale factor $\beta_{j}\left(=\mu_{j} / \alpha_{j}\right)$ depend on the values of $\mu_{j}$ and $\sigma_{j}$ assigned to parameter $\theta_{j}$. The shape factor $\alpha_{j}$ is only dependent on the corresponding coefficient of variation $\left(\mathrm{COV}_{j}\right)$. It is expected that damage will cause large deviations from these measured initial values in lower stories, but smaller deviations in higher stories; therefore, the following values of $\mathrm{COV}_{j}$ are proposed, for all damage states:

$\mathrm{COV}_{j}= \begin{cases}0.35 & \text { for } j=1, \ldots, 3 \\ 0.25 & \text { for } j=4, \ldots, 10\end{cases}$

The scale factor $\beta_{j}$ differs for each damage state; for a stage $\mathrm{S} k, \beta_{j}^{(S k)}$ is chosen such that the maximum a

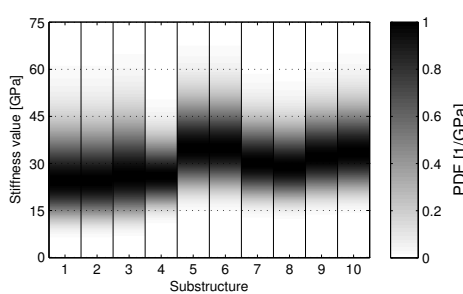

(a)

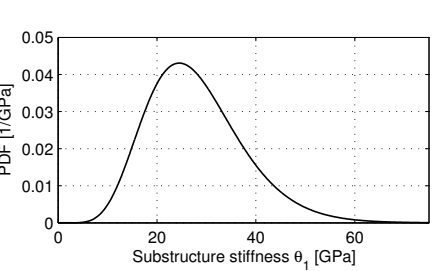

(b)
Figure 4: (a) Contour plot of the normalized marginal prior PDFs and (b) marginal prior PDF for substructure 1, for damage state S0.

priori point (i.e., the mode of the prior PDF) corresponds to the MAP estimate $\hat{\boldsymbol{\theta}}_{\mathrm{MAP}}^{(S(k-1))}$ of the previous damage stage $\mathbf{S}(k-1)$. This yields:

$\beta_{j}^{(S k)}=\hat{\theta}_{\mathrm{MAP}, j}^{(S(k-1))} \operatorname{COV}_{j}^{2}\left(1-\mathrm{COV}_{j}^{2}\right)^{-1}$

Figure 4 illustrates the prior PDF for damage state SO.

\subsubsection{Likelihood function}

For each damage stage, a fixed uncorrelated zeromean Gaussian prediction error is adopted:

$\boldsymbol{\eta}^{(S k)} \sim \mathcal{N}\left(\mathbf{0}, \boldsymbol{\Sigma}_{\boldsymbol{\eta}}^{(S k)}\right)$

where it is assumed that the covariance matrix $\Sigma_{\eta}^{(S k)}$ is known. In this test case, the prediction error $\boldsymbol{\eta}^{(S k)}$ represents the discrepancy between measured and computed eigenvalues and mode shapes:

$\boldsymbol{\eta}^{(S k)}=\left[\begin{array}{l}\boldsymbol{\eta}_{\lambda}^{(S k)} \\ \boldsymbol{\eta}_{\phi}^{(S k)}\end{array}\right]=\left[\ldots, \eta_{\lambda, r}^{(S k)}, \ldots, \eta_{\phi, r, \ell}^{(S k)}, \ldots\right]^{\mathrm{T}}$

where $r=1, \ldots, N_{m}$ and $\ell=1, \ldots, N_{o}$. The assumption of a zero mean value for $\boldsymbol{\eta}^{(S k)}$ corresponds to assuming that the computed values will on average be equal to the measured values. For the eigenvalues discrepancies $\eta_{\lambda, r}^{(S k)}$, it is assumed that the standard deviations are proportionate to the measured values:

$\eta_{\lambda, r}^{(S k)} \sim \mathcal{N}\left(0, c_{\lambda, r}^{2}\left(\tilde{\lambda}_{r}^{(S k)}\right)^{2}\right)$

In this way, the values of $c_{\lambda, r}$ can be interpreted as appointed coefficients of variation. For the mode shape components, a slightly different strategy is adopted in order to avoid assigning extremely small standard deviations to components with measured values close to zero. Instead, for each mode shape component $\ell$ of a mode $r$, the same standard deviation is assumed proportionate to the norm of mode shape $r$, such that:

$\eta_{\phi, r, \ell}^{(S k)} \sim \mathcal{N}\left(0, c_{\phi, r}^{2}\left\|\tilde{\phi}_{r}^{(S k)}\right\|^{2}\right)$

The values of $c_{\lambda, r}$ and $c_{\phi, r}$ reflect the magnitude of the combined modeling and measurement error. In this particular case, however, only limited information is available regarding the measurement error, in 
the form of observed variabilities of identified natural frequencies using different system identification methods and ambient vibration tests (Moaveni et al. 2011, Moaveni et al. ). Based on these studies and engineering judgment, the following values for $c_{\lambda, r}$ and $c_{\phi, r}$ are proposed for the three experimentally identified modes, for all damage states:

$\mathbf{c}_{\lambda}=\mathbf{c}_{\phi}=\left[\begin{array}{lll}0.069 & 0.150 & 0.100\end{array}\right]$

Using the expressions in Eqs. (5), (14) and (15), the likelihood function for a single data set $\tilde{\mathbf{d}}^{(S k)}$ can now be written as:

$$
\begin{aligned}
L\left(\boldsymbol{\theta} \mid \tilde{\mathbf{d}}^{(S k)}\right) & \propto \exp \left[-\frac{1}{2}\left(\boldsymbol{\eta}^{(S k)}\right)^{\mathrm{T}}\left(\boldsymbol{\Sigma}_{\boldsymbol{\eta}}^{(S k)}\right)^{-1}\left(\boldsymbol{\eta}^{(S k)}\right)\right] \\
& \propto \exp \left[-\frac{1}{2} J_{\mathrm{ML}}\left(\boldsymbol{\theta}, \tilde{\mathbf{d}}^{(S k)}\right)\right]
\end{aligned}
$$

where $J_{\mathrm{ML}}\left(\boldsymbol{\theta}, \tilde{\mathbf{d}}^{(S k)}\right)$ is the ML objective function (often also referred to as the misfit function):

$$
\begin{aligned}
J_{\mathrm{ML}}\left(\boldsymbol{\theta}, \tilde{\mathbf{d}}^{(S k)}\right) & =\sum_{r=1}^{N_{m}} \frac{1}{c_{\lambda, r}^{2}} \frac{\left(\tilde{\lambda}_{r}^{(S k)}-\lambda_{r}(\boldsymbol{\theta})\right)^{2}}{\left(\tilde{\lambda}_{r}^{(S k)}\right)^{2}} \\
& +\sum_{r=1}^{N_{m}} \frac{1}{c_{\phi, r}^{2}} \frac{\left\|\tilde{\boldsymbol{\phi}}_{r}^{(S k)}-\boldsymbol{\phi}_{r}(\boldsymbol{\theta})\right\|^{2}}{\left\|\tilde{\boldsymbol{\phi}}_{r}^{(S k)}\right\|^{2}}
\end{aligned}
$$

\subsubsection{MAP estimate and deterministic updating}

It is easily verified that maximizing a Gaussian likelihood function corresponds to solving an unregularized least squares problem. By maximizing the $(\log )$ posterior PDF (in order to find the Maximum A Posteriori or MAP estimate $\hat{\boldsymbol{\theta}}^{\mathrm{MAP}}$ ), the prior PDF is included into this scheme, naturally introducing a regularization term into the corresponding deterministic optimization problem.

For the seven-story test structure, the MAP objective function for a damage state $\mathrm{S} k$ is constructed as:

$J_{\mathrm{MAP}}\left(\boldsymbol{\theta}, \tilde{\mathbf{d}}^{(S k)}\right)=\frac{1}{2} J_{\mathrm{ML}}\left(\boldsymbol{\theta}, \tilde{\mathbf{d}}^{(S k)}\right)-\log p\left(\boldsymbol{\theta} ; \hat{\boldsymbol{\theta}}_{\mathrm{MAP}}^{(S(k-1))}\right)$

Elaborating the second term, i.e. the prior objective function $J_{\mathrm{MAPr}}^{(S k)}$, yields (up to a constant term):

$J_{\mathrm{MAPr}}^{(S k)}=\sum_{j=1}^{N_{\theta}}\left(\frac{\theta_{j}}{\beta_{j}^{(S k)}}+\left(1-\alpha_{j}\right) \log \theta_{j}\right)$

It is clear that the first term in the above equation corresponds to a weighted L1 regularization term, which encourages sparsity of the parameter vector such that only the most relevant parameters remain. The second

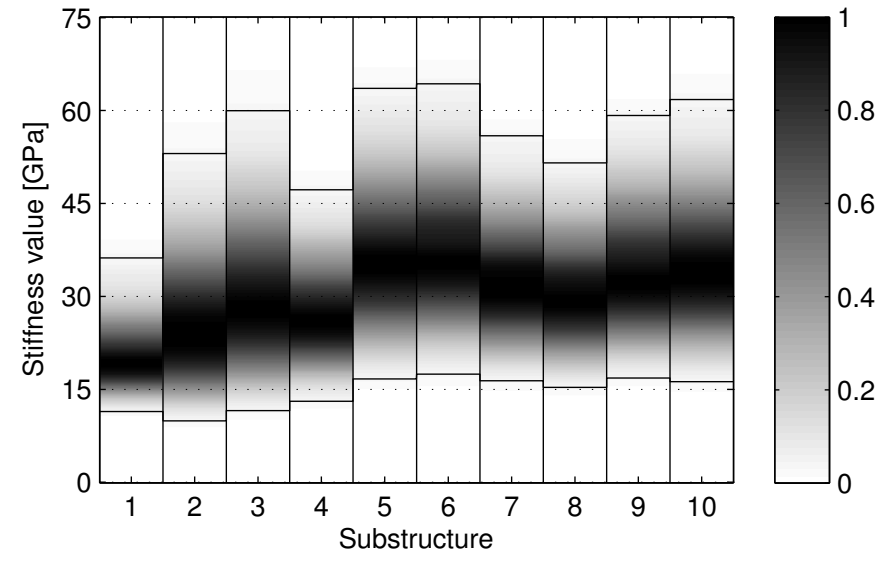

Figure 5: Contour plot and 99\% confidence bounds of the normalized marginal posterior PDFs for all substructures, for damage state S0.

term acts as a barrier function which enforces the constraint of positivity on the model parameters $\boldsymbol{\theta}$, as the factor $\left(1-\alpha_{j}\right)$ is here always negative and the corresponding second term therefore pushes the solution for $\theta_{j}$ away from zero in the positive direction. This clearly illustrates that the term $J_{\mathrm{MAPr}}^{(S k)}$ (or $-\log p(\boldsymbol{\theta})$ in general) can be interpreted as a regularization term which is based only on the available prior information and avoids having to revert to other standard regularization approaches. Furthermore, constraints contained in the prior information are automatically enforced.

\subsubsection{Results of the Bayesian updating scheme}

For each damage stage, the joint posterior PDF of the model parameters $\boldsymbol{\theta}$ was sampled using the Adaptive Metropolis-Hastings MCMC method (Haario et al. 2001). Several convergence measures (i.e. running mean values, running standard deviations and running correlation between samples) showed that for all damage states, convergence was reached after 200000 samples. The marginal posterior PDFs were obtained by kernel smoothing density estimation.

Results for damage state S0 The normalized marginal posterior PDFs for S0 are shown in Figure 5; Table 3 reports the corresponding MAP estimate, and the mean value, standard deviation and coefficient of variation for each of the marginal PDFs.

When examining the MAP-estimate and posterior mean values, it is clear that the effective stiffness in the undamaged state of the building was initially underestimated in most substructures, except for the bottom substructure 1, which shows a low value compared to the initial value $\theta_{1}^{\text {init }}$. Furthermore, among all the stiffness parameters, this bottom substructure stiffness is best identified from the data and prior information, as the COV is reduced from $35 \%$ to about $24 \%$. For the top seven substructures, the uncertainty is reduced only to a very limited extent below the prior $\mathrm{COV}$ of $25 \%$ : the posterior $\mathrm{COV}$-values range 
Table 3: Initial values, MAP estimates, posterior mean values $\mu$, standard deviations $\sigma$ and coefficients of variation $(\mathrm{COV})$ for S0.

\begin{tabular}{c|c|ccc}
\hline \hline $\begin{array}{c}\text { Sub- } \\
\text { structure }\end{array}$ & $\boldsymbol{\theta}^{\text {init }}$ & $\boldsymbol{\theta}^{\mathrm{MAP}}$ & $\begin{array}{c}\text { S0 }[\mathrm{GPa}] \\
\mu(\sigma)\end{array}$ & COV $[\%]$ \\
\hline 1 & 24.50 & 20.91 & $21.33(5.10)$ & 23.9 \\
2 & 24.50 & 24.83 & $27.41(8.77)$ & 32.0 \\
3 & 26.00 & 27.87 & $31.56(9.86)$ & 31.3 \\
4 & 26.00 & 27.79 & $28.07(6.75)$ & 24.0 \\
5 & 34.80 & 36.06 & $37.23(9.25)$ & 24.8 \\
6 & 34.80 & 32.56 & $38.12(9.19)$ & 24.1 \\
7 & 30.20 & 29.14 & $33.43(7.69)$ & 23.0 \\
8 & 28.90 & 31.53 & $31.42(7.26)$ & 23.1 \\
9 & 32.10 & 32.67 & $35.14(8.33)$ & 23.7 \\
10 & 33.50 & 33.12 & $36.05(8.93)$ & 24.8 \\
\hline \hline
\end{tabular}

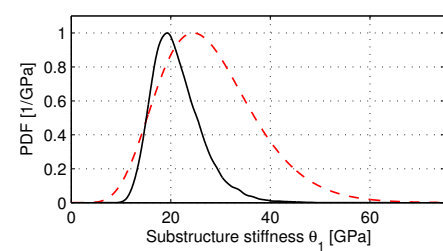

(a)

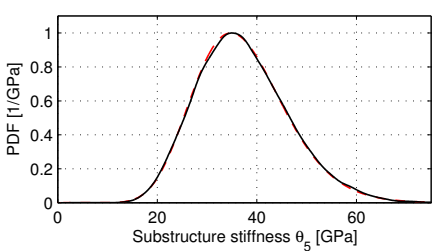

(b)
Figure 6: Normalized marginal prior PDF (dashed line) and posterior PDF (solid line) for (a) substructure 1 and (b) substructure 5, for damage state S0.

from $23 \%$ to $24.8 \%$. In Figure 6, the normalized prior and posterior marginal PDFs are compared for substructures 1 and 5, which immediately confirms these findings.

Results for damage states S1 to S4 In Figures 7a$7 \mathrm{~d}$, contour plots of the posterior marginal PDFs of the effective stiffness parameters $\boldsymbol{\theta}_{\mathrm{M}}$ are shown for damage states S1 to S4. The MAP-estimates (obtained through MCMC) of the stiffness values are compared in Figure 8 for all damage states, the corresponding values are reported in Table 4, together with the posterior marginal coefficients of variation.

The MAP stiffness values generally reduce as the damage increases, especially the stiffness in the bottom substructures - where the actual damage from the shake table tests is concentrated. The most drastic stiffness reduction occurs for substructure 1, where the MAP stiffness at $\mathrm{S} 4$ decreases to about $1 \mathrm{GPa}$ due to the very high level of damage. For some substructures, sometimes a small increase in MAP stiffness is found for a higher damage state, which is most likely caused by insensitivity of the model predictions to changes in these parameter values, resulting low identifiability. This is corroborated by the fact that the posterior uncertainty regarding these substructures remains largely the same over all damage states.

Overall, the lower part of the structure (substructures 1-3) shows a larger COV reduction compared to the top substructures, especially in states $\mathrm{S} 0$ and $\mathrm{S} 1$, and particularly for the bottom substructure, where the posterior $\mathrm{COV}$ is even reduced to about half of the prior COV in S4. These observations are most likely

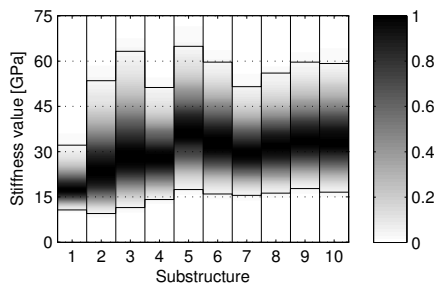

(a) $\mathrm{S} 1$

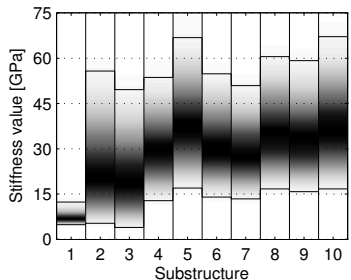

(c) $\mathrm{S} 3$

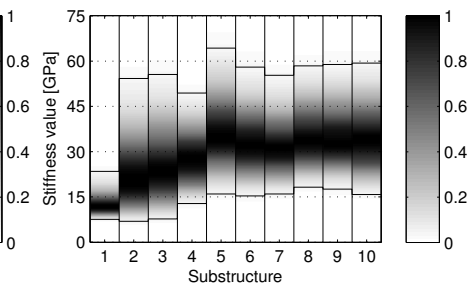

(b) $\mathrm{S} 2$

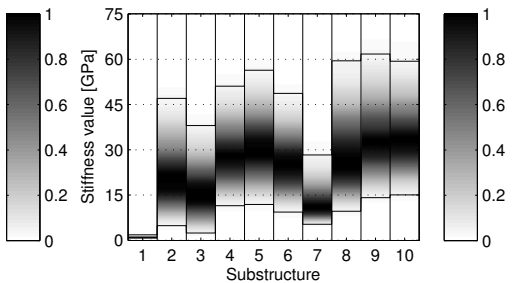

(d) $\mathrm{S} 4$

Figure 7: Contour plot and 99\% confidence bounds of the normalized marginal posterior PDFs for all substructures, for damage states S1 to S4.

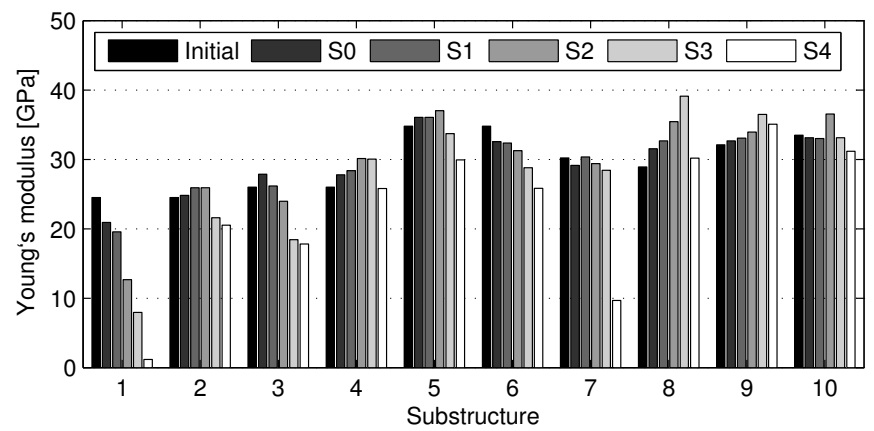

Figure 8: Initial stiffness values $\boldsymbol{\theta}^{\text {init }}$ and MAP stiffness values for all damage states.

explained by considering modal curvatures: firstly, the lower section of the structure is in any case subjected to higher modal curvatures, meaning the modal data are more sensitive to local stiffness changes and thus provide more information for the updating scheme in these areas. Moreover, structural damage results in an additional increase in modal curvature, explaining the substantial uncertainty reduction in the most damaged bottom substructure 1 . This also implies that, as the damage increases, the data become relatively less informative regarding substructures with less extensive damage. Examining the posterior COV-values for higher damage levels S3 and S4 confirms this statement: for substructures 2-10 the uncertainty no longer reduces, and sometimes even increases slightly due to this effect.

All these findings indicate that the available data are not always as informative regarding the chosen model parameters. This also implies that the available prior information plays an important role in the results obtained through the Bayesian inference scheme. In order to confirm these statements and to obtain more insight into the underlying causes of these findings, a detailed resolution and uncertainty analysis may be carried out, as presented in the next section. 
Table 4: MAP-values and coefficients of variation (COV) for the 10 substructure stiffnesses, for damage states $\mathrm{S} 1$ to $\mathrm{S} 4$.

\begin{tabular}{|c|c|c|c|c|c|c|c|c|}
\hline \multirow{2}{*}{$\begin{array}{l}\text { Sub- } \\
\text { struc- } \\
\text { ture }\end{array}$} & \multicolumn{2}{|c|}{$\overline{\mathrm{S} 1}$} & \multicolumn{2}{|c|}{$\overline{\mathrm{S} 2}$} & \multicolumn{2}{|c|}{$\overline{\text { S3 }}$} & \multicolumn{2}{|c|}{$\overline{\text { S4 }}$} \\
\hline & $\begin{array}{l}\text { MAP } \\
\text { [GPa] }\end{array}$ & $\begin{array}{c}\mathrm{COV} \\
{[\%]}\end{array}$ & $\begin{array}{l}\text { MAP } \\
{[\mathrm{GPa}]}\end{array}$ & $\begin{array}{c}\mathrm{COV} \\
{[\%]}\end{array}$ & $\begin{array}{l}\text { MAP } \\
\text { [GPa] }\end{array}$ & $\begin{array}{c}\mathrm{COV} \\
{[\%]}\end{array}$ & $\begin{array}{l}\text { MAP } \\
{[\mathrm{GPa}]}\end{array}$ & $\begin{array}{c}\mathrm{COV} \\
{[\%]}\end{array}$ \\
\hline 1 & 19.56 & 23.1 & 12.66 & 24.4 & 7.96 & 22.7 & 1.16 & 19.1 \\
\hline 2 & 25.92 & 33.0 & 25.92 & 38.3 & 21.61 & 40.1 & 20.53 & 37.7 \\
\hline 3 & 26.17 & 31.7 & 23.97 & 35.6 & 18.43 & 40.8 & 17.80 & 40.9 \\
\hline 4 & 28.36 & 24.3 & 30.12 & 25.0 & 30.04 & 25.9 & 25.81 & 27.0 \\
\hline 5 & 36.06 & 24.7 & 37.03 & 25.4 & 33.70 & 25.3 & 29.94 & 27.6 \\
\hline 6 & 32.36 & 24.3 & 31.27 & 24.6 & 28.80 & 24.9 & 25.82 & 29.0 \\
\hline 7 & 30.35 & 23.2 & 29.40 & 23.4 & 28.43 & 24.6 & 9.68 & 36.2 \\
\hline 8 & 32.68 & 23.2 & 35.44 & 22.5 & 39.10 & 24.0 & 30.18 & 32.7 \\
\hline 9 & 33.06 & 22.9 & 33.94 & 22.7 & 36.49 & 24.5 & 35.07 & 27.3 \\
\hline 10 & 33.01 & 24.0 & 36.53 & 24.3 & 33.13 & 25.4 & 31.18 & 25.8 \\
\hline
\end{tabular}

\section{RESOLUTION ANALYSIS}

The first step in a resolution analysis typically consists in determining quantities such as MAP estimates, posterior mean values and standard deviations, which yield basic insight into the resolution of the parameters. To obtain information regarding possible correlations between parameters, the prior and posterior covariance matrices, denoted as $S_{p r}$ and $S_{p o}$ respectively, may be calculated.

In order to obtain improved insight into the resolution of parameter combinations, several authors (Tarantola 2005, Duijndam 1988) propose to examine the solution of the following extended eigenvalue problem:

$\mathrm{S}_{\mathrm{po}} \mathrm{X}=\Lambda \mathrm{S}_{\mathrm{pr}} \mathrm{X}$

It can be shown that the eigenvectors in $\mathrm{X}$ correspond to mutually orthogonal directions in the parameter space ranked according to decreasing reduction from prior to posterior variance, when ranked according to increasing eigenvalue. Each eigenvalue gives a measure for the ratio of posterior to prior variance in the corresponding direction in the parameter space, which means that the eigenvector associated with the smallest eigenvalue corresponds to a direction in the parameter space that shows the largest reduction from prior to posterior variance. In other words, the values of the eigenvalues express the relative degree of the reduction from prior to posterior variance in the principal directions in the parameter space.

\subsection{Relation to information entropy}

As a measure of the information gained from the observations, the entropy discrepancy $\Delta h$ may be computed:

$\Delta h=h_{\mathrm{pr}}-h_{\mathrm{po}}$

It can be shown that, under certain asymptotic conditions (Papadimitriou et al. 2000, Papadimitriou 2004, Katafygiotis and Beck 1998), the entropy discrepancy can be approximated as:

$\Delta h \approx-\frac{1}{2} \log \operatorname{det}\left(\mathbf{S}_{\mathrm{pr}}^{-1} \mathbf{S}_{\mathrm{po}}\right)=-\frac{1}{2} \sum_{k=1}^{N_{\theta}} \log \lambda_{k}$ where $\lambda_{k}$ are the eigenvalues of the eigenvalue problem defined in Eq. (22). This means that by computing the values $d_{k}=-\frac{1}{2} \log \lambda_{k}$ corresponding to the eigenvectors (or directions in the parameter space) $\mathbf{X}_{k}$, the relative contribution of the different directions to the total resolution can be quantified.

\subsection{Resolution analysis for damage state SO of the seven-story test structure}

The posterior correlation coefficient matrix for the substructure stiffnesses is shown in Figure 9a for damage state $\mathrm{S} 0$, from which it can be deduced that, in contrast to the prior situation, the model parameters are a posteriori no longer independent variables. However, the correlations between the model parameters generally remain very limited, except for the bottom substructures where correlation coefficients of -0.42 are attained. Note that the occurring correlations are mostly negative, which is to be expected as contrasting stiffnesses (i.e. high in one and low in the other) in (adjacent) substructures would explain the data almost equally well.

In Figure 9b, the first and last two (normalized) eigenvectors or parameter combinations are shown, corresponding to the best and worst resolved directions in the parameter space. It is clear that the best resolved parameter combination contains predominantly the first substructure stiffness, whereas the two worst resolved directions contain all seven of the top substructure stiffness values. This is in very good agreement with the previously discussed results. Examining the eigenvalues associated with these eigenvectors shows that the total entropy reduction $\Delta h$ equals 1.21, of which a part of $\left(-1 / 2 \log \lambda_{1}=\right) 0.92$ or $76 \%$ is contributed by reduction in the direction $X_{1}$. Directions $X_{9}$ and $X_{10}$ together contribute a mere $0.04 \%$ to the total entropy reduction, confirming the results found above.

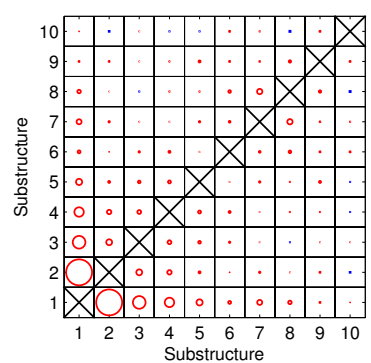

(a)

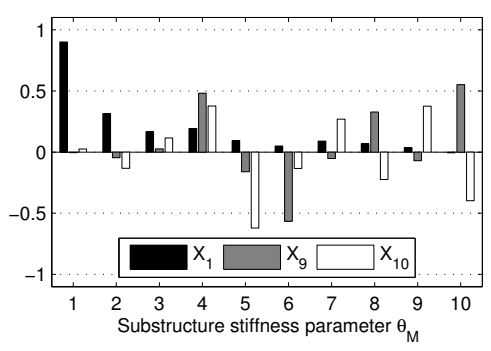

(b)
Figure 9: (a) Visualization of posterior correlation coefficient matrix where the relative size of the symbols represents the value of the negative ( 0 ) and positive $(\square)$ correlation coefficients, and (b) the best $\left(X_{1}\right)$ and two worst $\left(X_{9}\right.$ and $\left.X_{10}\right)$ resolved parameter combinations, for damage state $\mathrm{S} 0$. 
In this paper, Bayesian linear FE model updating is used for uncertainty quantification in the assessment of progressive damage in a seven-story reinforced concrete building slice subjected to seismic tests on the USCSD-NEES shake table. To this end, experimentally identified modal data obtained in five different damage states are employed.

Overall, the Bayesian approach succeeded in identifying the damage in the seven-story structure and in quantifying the corresponding uncertainties at all damage states. It was found that the data contain little information concerning the top stories of the building, as the uncertainty on the stiffness parameters representing this area could not be reduced through the observed data. This was confirmed by a detailed resolution analysis, which showed that parameter combinations containing the upper seven substructures were always least resolved by the available data. However, the lower substructures, and the bottom substructure 1 in particular, are well resolved by the data, most likely due to the higher damage level and higher modal curvatures in these areas of the structure.

These findings lead to the conclusion that for this structure, damage can be detected (SHM Level 1 (Rytter 1993)) effectively, but that for the purpose of reducing the uncertainty regarding damage quantification and localization (SHM levels 2-3) in the upper stories, more elaborate experimental data are desirable. This can be accomplished by increasing the number of mode shapes and/or measurement DOFs, or by including other types of modal data such as modal strains.

\section{REFERENCES}

Beck, J. \& L. Katafygiotis (1998). Updating models and their uncertainties. I: Bayesian statistical framework. ASCE Journal of Engineering Mechanics 124(4), 455461.

Ching, J. \& Y.-C. Chen (2007). Transitional Markov Chain Monte Carlo method for Bayesian model updating, model class selection, and model averaging. ASCE Journal of Engineering Mechanics 133(7), 816-832.

Duijndam, A. (1988). Bayesian estimation in seismic inversion. Part II: Uncertainty analysis. Geophysical Prospecting 36(8), 899-918.

Filippou, F. \& M. Constantinides (2004). Fedeaslab getting started guide and simulation examples. Technical report NEESgrid-2004-22.

Gamerman, D. (1997). Markov Chain Monte Carlo: stochastic simulation for Bayesian inference. London: Chapman \& Hall.

Haario, H., M. Laine, A. Mira, \& E. Saksman (2006). DRAM: Efficient adaptive MCMC. Statistics and Computing 16(4), 339-354.

Haario, H., E. Saksman, \& J. Tamminen (2001). An adaptive Metropolis algorithm. Bernouilli 7(2), 223-242.
Jaynes, E. (1957). Information theory and statistical mechanics. The Physical Review 106(4), 620-630.

Katafygiotis, L. \& J. Beck (1998). Updating models and their uncertainties. II: Model identifiability. ASCE Journal of Engineering Mechanics 124(4), 463-467.

Moaveni, B., A. Barbosa, J. Conte, \& F. Hemez. Uncertainty analysis of system identification results obtained for a seven story building slice tested on the UCSDNEES shake table. Structural Control and Health Monitoring. Under review.

Moaveni, B., A. Barbosa, J. Conte, \& F. Hemez (2007, February). Uncertainty analysis of modal parameters obtained from three system identification methods. In Proceedings of IMAC-XXV, International Conference on Modal Analysis, Orlando, Florida, USA.

Moaveni, B., J. Conte, \& F. Hemez (2009). Uncertainty and sensitivity analysis of damage identification results obtained using finite element model updating. ComputerAided Civil and Infrastructure Engineering 24(5), 320334.

Moaveni, B., X. He, J. Conte, \& J. Restrepo (2010). Damage identification study of a seven-story full-scale building slice tested on the UCSD-NEES shake table. Structural Safety 32, 347-356.

Moaveni, B., X. He, J. Conte, J. Restrepo, \& M. Panagiotou (2011). System identification study of a 7-story fullscale building slice tested on the USCD-NEES shake table. ASCE Journal of Engineering Mechanics 137(6), 705-717.

Papadimitriou, C. (2004). Optimal sensor placement for parametric identification of structural systems. Journal of Sound and Vibration 278, 923-947.

Papadimitriou, C., J. Beck, \& S. Au (2000). Entropy-based optimal sensor location for structural model updating. Journal of Vibration and Control 6(5), 781-800.

Papadimitriou, C., J. Beck, \& L. Katafygiotis (1997). Asymptotic expansions for reliability and moments of uncertain systems. ASCE Journal of Engineering Mechanics 123(12), 1219-1229.

Rytter, A. (1993). Vibration based inspection of civil engineering structures. Ph. D. thesis, Aalborg University.

Simoen, E., B. Moaveni, J.P. Conte, \& G. Lombaert (2013). Uncertainty quantification in the assessment of progressive damage in a seven-story full-scale building slice. ASCE Journal of Engineering Mechanics. Published online. http://dx.doi.org/10.1061/(ASCE)EM.19437889.0000610

Soize, C. (2003, May). Probabilités et modélisation des incertitudes: éléments de base et concepts fondamentaux. Handed out at the séminaire de formation de l'école doctorale MODES, Paris.

Soize, C. (2008). Construction of probability distributions in high dimensions using the maximum entropy principle: applications to stochastic processes, random fields and random matrices. International Journal for Numerical Methods in Engineering 75, 1583-1611.

Tarantola, A. (2005). Inverse problem theory and methods for model parameter estimation. Philadelphia, USA: SIAM. 